\title{
CO in NGC 4314: The Detection of Inflow Along the Bar
}

\author{
G. Fritz Benedict \\ McDonald Observatory, University of Texas, Austin, TX 78712
}

Beverly J. Smith

IPAC, California Institute of Technology, Pasadena, CA 91125

J. D. P. Kenney

Department of Astronomy, Yale University, New Haven, CT 06511

\begin{abstract}
We present $\mathrm{CO}(1-0)$ interferometer data with $22^{\prime \prime} 3 \times 22^{\prime \prime 2}$ spatial resolution and $13 \mathrm{~km} \mathrm{~s}^{-1}$ velocity resolution acquired at the $O$ wens Valley Radio Observatory. These data reveal a clumpy circumnuclear ring of gas. We also find a central peak of $\mathrm{CO}$ within $2^{\prime \prime}$ of the optical center. Deviations from circular motion are seen where the dust lanes along the leading edge of the bar intersect the nuclear ring, consistent with gas inflow along the bar. H $\alpha$ maps of this galaxy show bright HII regions near the ends of this inflow region, indicating triggering of star formation by the inflow.
\end{abstract}

\section{Introduction}

We discuss CO observations of the nuclear region of NGC 4314. This is a summary of a more detailed analysis (including naturally-weighted reductions showing $\mathrm{CO}$ along much of the length of the primary stellar bar) to appear elsewhere (Benedict, Smith, \& Kenney 1995).

\section{Observations}

We observed NGC 4314 in the CO (1-0) line using the Owens Valley Radio Observatory (OVRO) millimeter-wave interferometer (Padin et al. 1991) in five configurations between April and June 1991. At that time OVRO consisted of a three element array with $10.4 \mathrm{~m}$ dishes $\left(\mathrm{HPBW}=65^{\prime \prime}\right)$ and SIS receivers. The $325 \mathrm{MHz}$ receivers give $13 \mathrm{~km} \mathrm{~s}^{-1}$ resolution and an instantaneous bandpass of $416 \mathrm{~km} \mathrm{~s}^{-1}$.

\section{Morphology}

Figure 1 presents the uniformly weighted CO intensity map. We confirm the CO ring first seen by Combes et al. (1992). The overall impression is of an 


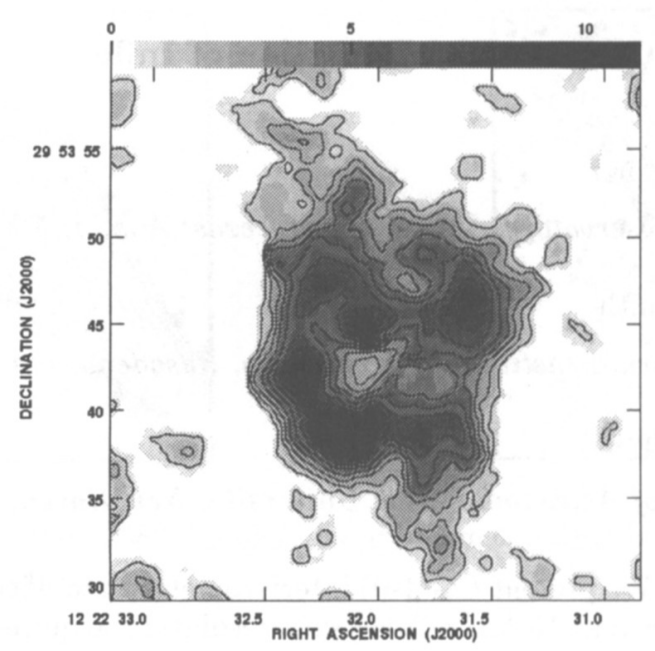

Figure 1. CO contours superposed on the gray scale CO map. The beam size is $2 . \prime 3 \times 2 . \prime 2$. The contour levels are $0.1,0.2, \ldots, 1.0$ times the peak intensity of $1.3 \mathrm{Jy}_{\text {beam }}{ }^{-1} \mathrm{~km} \mathrm{~s}^{-1}$. The $3 \sigma$ level is $0.153 \mathrm{Jy}$ beam $^{-1} \mathrm{~km} \mathrm{~s}^{-1}$, slightly higher than the lowest plotted contour.

incomplete, clumpy ring with an off-center minimum. There are five clumps of emission along the ring, separated by gaps. In addition to the clumps in the ring, we find strong $\mathrm{CO}$ emission within $2^{\prime \prime}$ of the optical center.

\section{Kinematics and Inflow Along the Bar}

From the naturally-weighted velocity field we have determined the systemic velocity $\mathrm{V}_{\text {sys }}=983 \mathrm{~km} \mathrm{~s}^{-1}$, the dynamical center of the galaxy, the position angle of the line of nodes $\phi=115^{\circ}$, the inclination $\mathrm{i}=23^{\circ}$, and a rotation curve $\mathrm{V}(\mathrm{R})$. We used the iterative technique of Puche et al. (1991), and included only data within $45^{\circ}$ of the major axis.

Dust lanes in the primary stellar bar which approach and intersect the nuclear ring were discussed at length in Benedict et al. (1992). Their analysis of the CO data of Garcia-Barreto et al. (1991) provided a marginal detection of non-circular motions associated with these dust lanes. To study these motions in more detail, we obtain a velocity residual map (Figure 2) by producing a model galaxy velocity field from our rotation curve and subtracting it from the observed $\mathrm{CO}$ velocity field.

If the southwest side of the galaxy is closest to us, and the dust lanes are on the leading edge of the bar, then the radial velocity deviations seen in Figure 2 imply inflow. Note that the inflow rates gradually decrease as the nuclear ring is approached and crossed. An inward flow along the leading edge of a bar is predicted by various models (Athanassoula 1992) and confirmed in Figure 2. The 


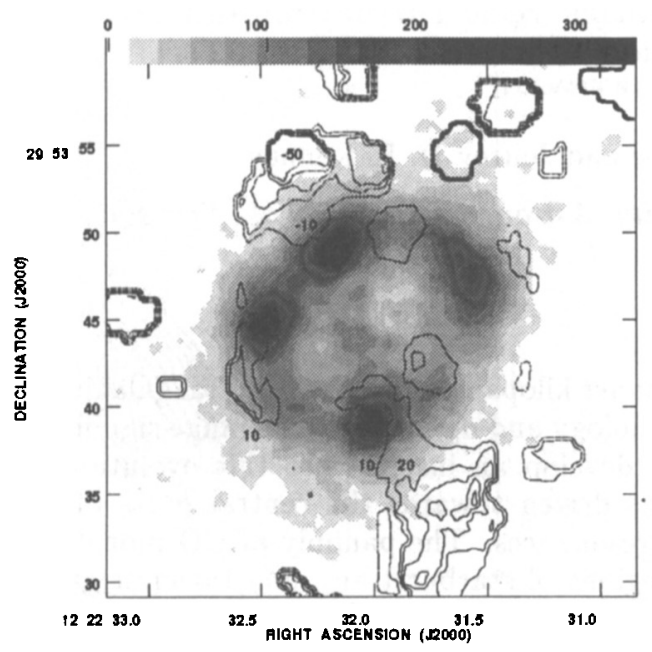

Figure 2. The natural weight $\mathrm{CO}$ velocity residual map superposed on $\mathrm{H} \alpha+[\mathrm{NII}]$ from Pogge (1989). Velocities in $\mathrm{km} \mathrm{s}^{-1}$. The gray scale is encoded to represent the strongest $\mathrm{H} \alpha+[\mathrm{N} \mathrm{II}]$ emission as black. Note that the deepest incursions of the decelerating gas terminate in close proximity to large HII regions.

magnitudes of these deviations (corrected for inclination) are $+90 \mathrm{~km} \mathrm{~s}^{-1}$ to the $\mathrm{SW}$ and $-90 \mathrm{~km} \mathrm{~s}^{-1}$ to the NE. Major $\mathrm{H} \alpha$ sources are seen (Pogge 1989) within $1^{\prime \prime}$ of the terminus of each merge site. This suggests that gas flowing inward along the bar collides with gas already in the ring, triggering star formation.

\section{References}

Athanassoula, E. 1992, MNRAS, 259, 345

Benedict, G. F., Higdon, J. L., Tollestrup, E. V., Hahn, J., \& Harvey, P. M. 1992, AJ, 103, 757

Benedict, G. F., Smith, B. J., \& Kenney, J. D. P. 1995, in preparation

Combes, F., Gerin, A., Nakai, N., Kawabe, R., \& Shaw, M. A. 1992, A\&A, 259, $\mathrm{L} 27$

Garcia-Barreto, J. A., Downes, D., Combes, F., Gerin, M., Magri, C., Carrasco, L., \& Cruz-Gonzalez, I. 1991, A\&A, 244, 257

Padin, S. et al. 1991, PASP, 103, 461

Pogge, R. W. 1989, ApJS, 71, 433

Puche, D., Carignan, C., \& Wainscoat, R. J. 1991, AJ, 101, 447 